\title{
Temporal evaluation of secondary user interference to primary user in cognitive radio networks
}

\begin{abstract}
In this paper, the quantity of interference on the primary user (PU) caused by the secondary user (SU) is investigated. An underlay spectrum sharing network is considered, where the different scenario of PU activity is modeled based on the duty cycle of PU channel. PU channel is classified into several activities according to the application of primary services. The interference to PU is observed in two scenarios, which are $40 \%$ and $65 \%$ utilization of the SU channel. The effect of interference to PU data rate also analyzed. Simulation results shown that as the PU channel become more congested, the quantity of interference of PU and probability of interference become increase. PU channel performance in term of data rate will improve as the shorter transmission time of SU.
\end{abstract}

Keyword: Cognitive radio; Duty cycle; Dynamic spectrum access; Quantity of interference; SU access time 